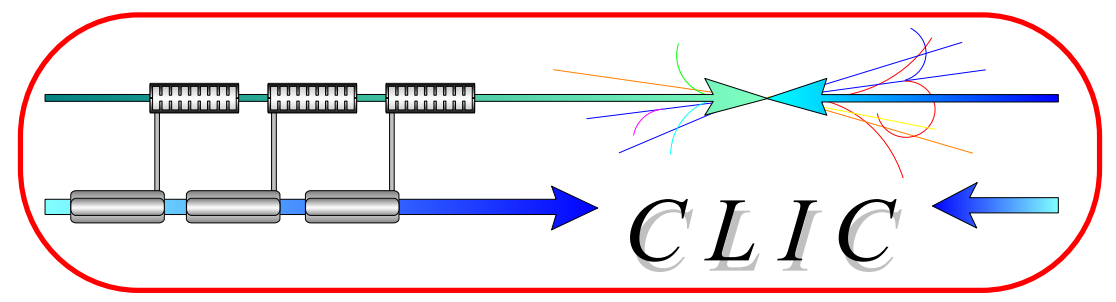

CLIC-NOTE-725

\title{
MATERIAL SELECTION AND CHARACTERIZATION FOR HIGH GRADIENT RF APPLICATIONS
}

\author{
G. Arnau-Izquierdo, S. Calatroni, S. Heikkinen, T. Ramsvik, S. Sgobba, M. Taborelli, W. Wuensch
}

\begin{abstract}
The selection of candidate materials for the accelerating cavities of the Compact Linear Collider (CLIC) is carried out in parallel with high power RF testing. The maximum DC breakdown field of copper, copper alloys, refractory metals, aluminium and titanium have been measured with a dedicated setup. Higher maximum fields are obtained for refractory metals and for titanium, which exhibits, however, important damages after conditioning. Fatigue behaviour of copper alloys has been studied for surface and bulk by pulsed laser irradiation and ultrasonic excitation, respectively. The selected copper alloys show consistently higher fatigue resistance than copper in both experiments. In order to obtain the best local properties in the device a possible solution is a bi-metallic assembly. Junctions of molybdenum and copper-zirconium UNS C15000 alloy, achieved by HIP (Hot Isostatic Pressing) diffusion bonding or explosion bonding were evaluated for their mechanical strength. The reliability of the results obtained with both techniques should be improved. Testing in DC and radiofrequency (RF) is continued in order to select materials for a bi-metal exhibiting superior properties with respect to the combination C15000-Mo.
\end{abstract}

Presented at: PAC07, Albuquerque, New Mexico, USA, June 25-29, 2007

Geneva, Switzerland

(June 2007) 


\title{
MATERIAL SELECTION AND CHARACTERIZATION FOR HIGH GRADIENT RF APPLICATIONS
}

\author{
G.Arnau-Izquierdo, S.Calatroni, S.Heikkinen, T.Ramsvik, S.Sgobba, M.Taborelli, W.Wuensch, \\ CERN, Geneva, Switzerland
}

\begin{abstract}
The selection of candidate materials for the accelerating cavities of the Compact Linear Collider (CLIC) is carried out in parallel with high power RF testing. The maximum DC breakdown field of copper, copper alloys, refractory metals, aluminium and titanium have been measured with a dedicated setup. Higher maximum fields are obtained for refractory metals and for titanium, which exhibits, however, important damages after conditioning. Fatigue behaviour of copper alloys has been studied for surface and bulk by pulsed laser irradiation and ultrasonic excitation, respectively. The selected copper alloys show consistently higher fatigue resistance than copper in both experiments. In order to obtain the best local properties in the device a possible solution is a bi-metallic assembly. Junctions of molybdenum and copper-zirconium UNS C15000 alloy, achieved by HIP (Hot Isostatic Pressing) diffusion bonding or explosion bonding were evaluated for their mechanical strength. The reliability of the results obtained with both techniques should be improved. Testing in DC and radiofrequency (RF) is continued in order to select materials for a bi-metal exhibiting superior properties with respect to the combination C15000-Mo.
\end{abstract}

\section{INTRODUCTION}

Particle accelerators of the future generation, as CLIC (Compact Linear Collider), are extremely demanding in terms of material properties for the accelerating structures. The TeV energy range can be obtained in machines of reasonable length only by applying accelerating field gradients of the order of some $100 \mathrm{MV} / \mathrm{m}$. The fields on the material surfaces will rise up to $300 \mathrm{MV} / \mathrm{m}$ and provoke electrical breakdown events, limiting the integrated luminosity and inducing irreversible damages on the geometry of the accelerating structures. Moreover, the high power RF pulses induce local heating and hence thermal fatigue on the surface of the accelerating structures. This will increase surface roughness and finally limit the lifetime of the structures. For the CLIC project two strategies in parallel are presently pursued. The more classical route consists in applying copper and copper alloys, which provide high electrical conductivity. An alternative approach is based on the application of refractory metals in the regions of highest electric fields, since they have shown a better performance at least in the high breakdown rate regime in RF [1] and DC [2]. High electrical conductivity materials must still be used in the highest magnetic field regions to preserve machine efficiency. Reliable methods to join the two materials in a bi-metallic system are being developed. The results of the characterization of copper and copper alloys from the point of view of breakdown and fatigue resistance are presented in the following, together with the mechanical tests performed on the bi-metal made of copper alloy and refractory metal.

\section{MATERIAL TESTING}

The materials selected for the fatigue and breakdown resistance are OFE copper (UNS C10100), the precipitation hardened alloy copper-zirconium $(\mathrm{CuZr})$ UNS C15000 (0.13-0.20\% wt of $\mathrm{Zr})$ and the Oxide Dispersion Strengthened (ODS) copper Glidcop ${ }^{\circledR}$ AL-15 (C15715) $\left(0.15 \%\right.$ wt $\left.\mathrm{Al}_{2} \mathrm{O}_{3}\right)$. They are chosen for their relatively high electrical conductivity compared to other copper alloys and superior mechanical properties with respect to OFE-copper, as shown in table 1. The tempers of fatigue test samples are also shown in table 1. For the breakdown resistance measurements the surfaces are as rolled for OFE copper and milled for the two alloys. All samples were cleaned by detergent, following the procedure for parts to be used in ultra high vacuum, before insertion in the measuring system. The $\mathrm{CuZr}$ for explosion bonding had the same properties mentioned in table 1. The molybdenum sheets used for explosion bonding are made of $99.97 \%$ pure molybdenum and are sintered, hot rolled and stress-relieved. The molybdenum rod used for Hot Isostatic Pressing (HIP) joining has purity better than $99.97 \%$ and is forged.

The DC breakdown field is measured in an apparatus described in details in ref [3]. A high voltage up to $15 \mathrm{kV}$ is applied to a gap junction by connecting it to a charged capacitor. A micro-positioning system enables to regulate the distance with micron accuracy between a hemispherical tip of $2.3 \mathrm{~mm}$ diameter, which represents the anode, and a plane cathode of $1 \mathrm{~mm}$ to $1.5 \mathrm{~mm}$ thickness. Both electrodes are made of the same material. The charge on the capacitor is measured before and after connecting it to the gap junction. The breakdown field is the maximum stable field, which can be applied to the gap junction without abruptly discharging the capacitor. The breakdown fields of OFE copper, CuZr and Glidcop ${ }^{\circledR}$ AL15 are compared in figure 1 , which shows the evolution of the breakdown field as a function of the number of breakdown events. In all cases saturation is reached after some 10 cycles, showing that the conditioning phase is very rapid. The field stabilizes about at the same level for the three materials and an average over 3 measurements for each material gives the following breakdown field and standard deviation: $151 \pm 39 \mathrm{MV} / \mathrm{m}$ for OFE copper, $120 \pm 26 \mathrm{MV} / \mathrm{m}$ for $\mathrm{CuZr}$ and $114 \pm 7 \mathrm{MV} / \mathrm{m}$ for Glidcop ${ }^{\circledR}$. Therefore there is no statistically significant difference between the breakdown fields. 

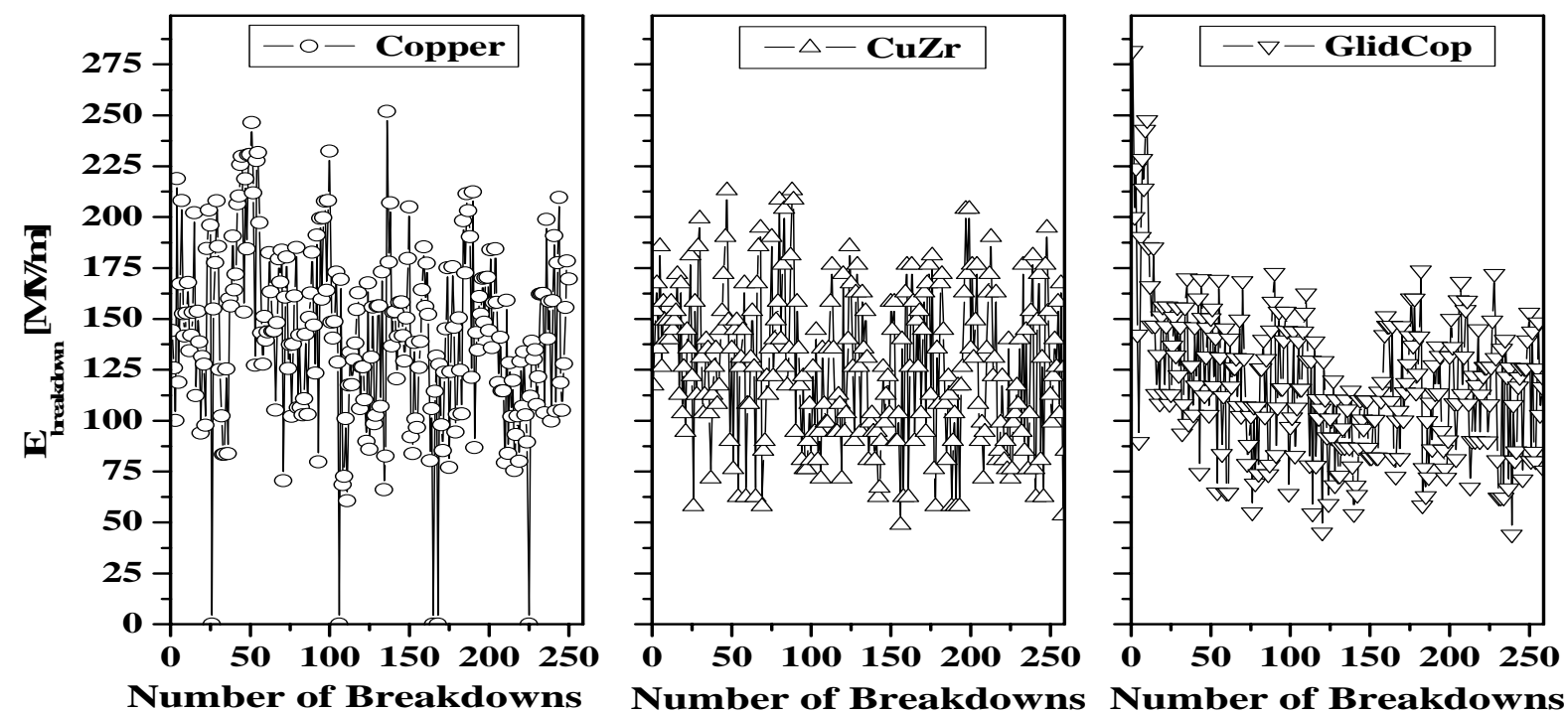

Figure 1: Comparison of the conditioning in DC breakdown experiment for OFE-Copper, CuZr, and Glidcop AL15

This measurement in DC does not provide directly the values of fields which can be applied also in the RF case. However, since it is well accepted that the mechanism of breakdown in DC and RF is initiated by field emission currents, we expect the ranking of material performance to be the same in DC and RF. The influence of the surface treatment is demonstrated by the comparatively slow conditioning behaviour of an electro discharge machined (EDM) CuZr surface, where about 50 breakdown events are necessary to reach the saturation value of the breakdown field. The final value is the same $(113 \pm 1 \mathrm{MV} / \mathrm{m})$ as for the milled surface. The difference in conditioning behaviour is reproducible over various spots on the same sample. The roughness values are $\mathrm{Ra}=0.21 \pm 0.01 \mu \mathrm{m}$ and $3.20 \pm 0.24 \mu \mathrm{m}$ for milled and EDM machined surface, respectively. Surface analysis shows that the cleanliness state of the various surface is identical. The difference can be due to a peculiarity of the EDM surface finishing or to the roughness.

Experiments to measure the fatigue resistance through high power RF pulses are in progress. As an alternative a similar cycle of pure compression through a temperature increase $\Delta \mathrm{T}$ in a depth comparable with the skin depth of $10-30 \mathrm{GHz} \mathrm{RF}$ can be induced by excimer laser $(308 \mathrm{~nm})$ pulses of comparable duration (40 ns) [4]. For each $\Delta \mathrm{T}$, which is proportional to the pulse energy density, the number of laser pulses inducing a given surface roughness $\mathrm{Ra}$ is monitored, as shown in figure 2 . For more clarity the values given in figure 2 are the amplitude of RF magnetic field which would induce the same stress as the $\Delta \mathrm{T}$ and are normalized to the target value for CLIC. Due to the limited repetition rate of the laser, such measurements are possible only up to $10^{7}$ cycles compared to the estimated CLIC lifetime of $2 * 10^{10}$ pulses. The data can be extended to a large number of cycles by using ultrasonic fatigue test specimens, excited at $24 \mathrm{kHz}$ in sinusoidal reversed tension-compression cycles [5]. In this case the fatigue resistance of the bulk material is measured by driving the specimens up to fracture. As it is visible in figure $2 \mathrm{CuZr}$ is superior to OFE copper, can withstand a larger number of cycles for the equivalent RF magnetic field and gives a larger margin above the CLIC target value. Hot extruded Glidcop ${ }^{\circledR}$ AL-15 can withstand a slightly lower number of cycles. The results are interpreted in terms of the tempers as reported in table 1 . Since for annealed OFE copper the tensile strength would be strongly reduced, and the same is expected to occur for the resistance to fatigue, an annealed temper would no longer fulfil the requirements for the machine lifetime. Also for $\mathrm{CuZr}$ the mechanical properties will be degraded by an inappropriate thermal treatment.

Table 1: Material properties of the specimens used for fatigue and breakdown resistance tests (IACS= International Annealed Copper Standard)

\begin{tabular}{|c|c|c|c|}
\hline $\begin{array}{c}\text { Material } \\
\text { UNS C }\end{array}$ & $\begin{array}{c}\text { Electrical } \\
\text { conduct. } \\
\text { (IACS\%) }\end{array}$ & $\begin{array}{c}\text { Temper } \\
\text { state }\end{array}$ & $\begin{array}{c}\text { Tensile } \\
\text { Strength } \\
\text { (MPa) }\end{array}$ \\
\hline $\begin{array}{c}\text { Cu-OFE } \\
(\mathrm{C} 10100)\end{array}$ & $101 \%$ & $\begin{array}{c}\text { cold worked } \\
\text { to half hard }\end{array}$ & $240-280$ \\
\hline $\begin{array}{c}\text { GlidCop } \\
\text { Al-15 } \\
(\mathrm{C} 15715)\end{array}$ & $90 \%$ & hot extruded & 393 \\
\hline $\begin{array}{c}\text { CuZr } \\
(\mathrm{C} 15000)\end{array}$ & $93 \%$ & $\begin{array}{c}40 \% \text { cold } \\
\text { worked and } \\
\text { aged }\end{array}$ & 340 \\
\hline
\end{tabular}

The advantage of Glidcop ${ }^{\circledR}$ is that it maintains the yield strength of its hot extruded state even after heating up to $800^{\circ} \mathrm{C}$ for $1 \mathrm{~h}$. Machining tests to verify the possibility to achieve high precision, as required for the accelerating structures on Glidcop ${ }^{\circledR}$ are foreseen. These fatigue tests were done up-to-fracture. For CLIC structures an increase in surface roughness would cause failure of the component through a runaway 
process. First attempts in studying the real damage criteria suggest approximately $10-20 \%$ lower stress amplitudes than the up-to-fracture values.

Several techniques can be envisaged to join a refractory metal to copper or copper alloy, as brazing and diffusion bonding by HIP. Two possible solutions to cope with the negative effect of thermal treatment on the mechanical properties of the copper based component consist in using Glidcop ${ }^{\mathbb{B}}$ or joining through a method which does not involve stay at critical temperatures. Following this rationale sheets of molybdenum of $1 \mathrm{~mm}$ to $3 \mathrm{~mm}$ thickness and $120 \mathrm{~mm}$ x $50 \mathrm{~mm}$ area were joined by explosion bonding to $\mathrm{CuZr} 40 \%$ cold worked and precipitation hardened. Samples for shear strength and tensile strength testing in a modified configuration with respect to references $[6,7]$ were machined from the resulting bi-metal parts. The results are listed in table 2. The shear strength values are comparable to the tensile strength of the weakest material in the junction, whereas the pull strength value is lower.

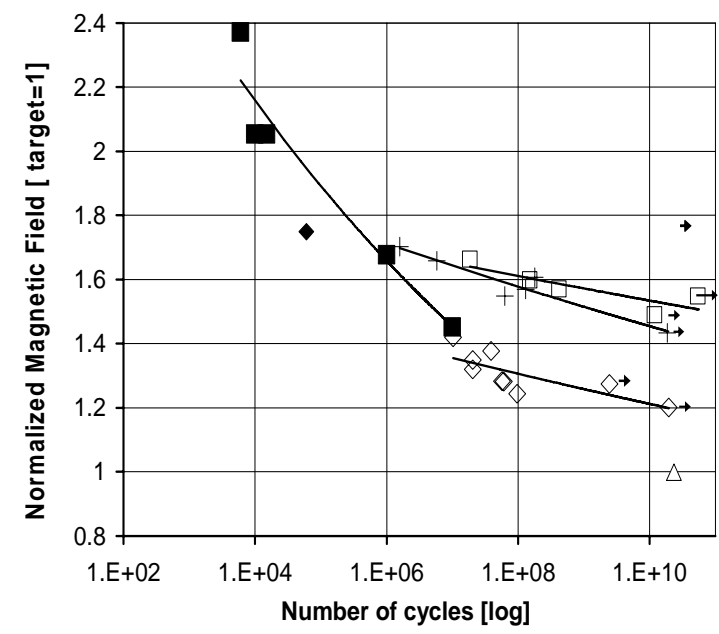

Figure2: Fatigue curve for OFE-copper (full diamond, laser; empty diamonds, ultrasound), $\mathrm{CuZr}$ (full squares, laser; empty squares, ultrasound) and Glidcop (crosses, ultrasound). Arrows indicate samples which did not fracture. Materials are in the state as in table 1 . The vertical axis is the equivalent amplitude of the RF magnetic field corresponding to the applied stress amplitude. It is normalized to 1 corresponding to the target value (triangle) for a CLIC design with $\Delta \mathrm{T}=56 \mathrm{~K}$.

Fracture always occurs in the molybdenum part, in particular in a region of the material close to the interface which is affected by the bonding process. The fracture surface presents a wavy corrugation. Hardness profiles across the interface indicate that changes in the mechanical properties are observed only in a region of $0.1 \mathrm{~mm}$ close to the interface. The tensile strength of the $\mathrm{CuZr}$ alloy measured after bonding is unaffected by the process $(358 \mathrm{MPa})$. For the moment only bonding of flat sheets has been carried out and a development is needed for different shapes. For the geometry of the irises of accelerating structures diffusion bonding by HIP offers the possibility to join in a cylindrical configuration a molybdenum core surrounded by $\mathrm{CuZr}$. A molybdenum rod of $8.6 \mathrm{~mm}$ diameter and $300 \mathrm{~mm}$ length has been joined to the surrounding $\mathrm{CuZr}$ of a diameter of $87 \mathrm{~mm}$. The results for the shear and tensile strength are given in table 2 (average values). For some samples significant striction can be observed, although one over 4 tensile strength specimens failed at zero stress (excluded from the average). This might be ascribed to an imperfect cleaning and smoothing of the joint faces before bonding. Trials with more accurate surface preparation are in progress. On the other hand, the shear strength of the interface $(165 \mathrm{MPa})$ is higher than the one reported for pure $\mathrm{Cu}(100 \mathrm{MPa})$ and close to the measured tensile strength of the $\mathrm{CuZr}$ matrix (199 MPa). The most important mechanical effort on the bi-metal material occurs during machining. Therefore, as ultimate test it has been proved that the junctions are sufficiently solid to resist milling of irislike shapes crossing the interface between the two metals. The test has been positive for both joining methods for parts including 3 to 6 irises of a typical structure.

Table2: Mechanical properties of the $\mathrm{Mo} / \mathrm{CuZr}$ joints by explosion bonding and HIP diffusion bonding.

\begin{tabular}{|c|c|c|}
\hline $\begin{array}{c}\text { Sample/ } \\
\text { strength (MPa) }\end{array}$ & $\begin{array}{c}\text { Explosion } \\
\text { 2mm Mo sheet }\end{array}$ & HIP rod \\
\hline Shear strength & $227-309$ & $165 \pm 9$ \\
\hline Pull strength & 77 & $175 \pm 18$ \\
\hline
\end{tabular}

\section{Aknowledgments}

We thank R.Stefanovich from the Belarus State Scientific and Production Powder Metallurgy Concern for the explosion bonding samples and P. Siitonen (METSO Powdermet Oy) for the HIP samples.

\section{REFERENCES}

[1] C. Achard, H. Braun, S. Döbert, I. Syratchev, M. Taborelli, I. Wilson, W. Wuensch, CLIC note 569 (CERN, Geneva, Switzerland 2003).

[2] T.Ramsvik. S.Calatroni, A.Reginelli, M.Taborelli. EPAC'06, Edinburg, July 2006, p.777 http://www.jacow.org

[3] M. Kildemo, Nucl. Instrum. Methods Phys. Res. A 530, (2004), 596.

[4] S. Calatroni et al EPAC'04, Lucerne, July 2004, p.557, http://www.jacow.org

[5] S.T.Heikkinen, S.Calatroni, H.Neupert and W.Wuensch, EPAC'06 Edinburg, June 2006 p. 858 http://www.jacow.org

[6] ASTM B 898 for shear test

[7] MIL-J-24445 A for tensile test 